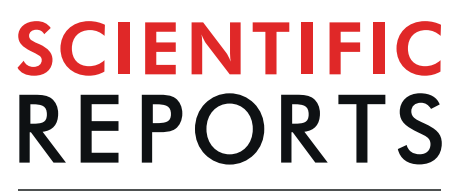

natureresearch

\title{
Islet-1 synergizes with Gon5 to promote MSC differentiation into cardiomyocytes
}

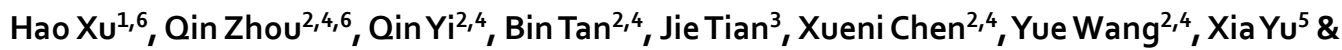 \\ Jing $\mathrm{Zhu}^{2,4^{*}}$
}

Mesenchymal stem cells (MSCs) specifically differentiate into cardiomyocytes as a potential way to reverse myocardial injury diseases, and uncovering this differentiation mechanism is immensely important. We have previously shown that histone acetylation/methylation and DNA methylation are involved in MSC differentiation into cardiomyocytes induced by islet-1. These modifications regulate cardiac-specific genes by interacting with each other in the promoter regions of these genes, but the molecular mechanism of these interactions remains unknown. In this study, we found that the key enzymes that regulate GATA4/Nkx2.5 expression are Gcn5/HDAC1, G9A, and DNMT-1. When $\alpha$-methylene- $\gamma$-butyrolactone 3 (MB-3) was used to inhibit Gcn5 expression, we observed that the interactions among these key enzymes in the GATA4/Nkx2.5 promoters were blocked, and MSCs could not be induced into cardiomyocytes. Our results indicated that islet- 1 could induce $\mathrm{Gcn} 5$ binding to GATA4/Nkx2.5 promoter regions and induce the interactions among Gcn5, HDAC1, G9A and DNMT-1, which upregulated GATA4/Nkx2.5 expression and promoted MSC differentiation into cardiomyocytes.

Myocardial injury diseases have always been among the highest lethality diseases, primarily due to myocardial cells having no self-renewal ability. Mesenchymal stem cells (MSCs) have been a hot global research topic because of their multiple differentiation potential ${ }^{1-4}$, and they can specifically differentiate into cardiomyocytes ${ }^{5-7}$, which could potentially help cure myocardial injury diseases. Many researchers have successfully induced MSC differentiation into cardiomyocytes through different methods ${ }^{8-10}$, but the molecular mechanism of differentiation is not clear, which results in low induction efficiency and limits the clinical application of MSCs. In our previous research, we overexpressed islet-1 and successfully induced MSC differentiation into cardiomyocyte-like cells that possess cardiac electrophysiological properties. Furthermore, we investigated the molecular mechanism and found that histone modifications and DNA methylation are very important for MSC differentiation; these epigenetic modifications interact with each other during MSC differentiation into cardiomyocytes ${ }^{11,12}$. However, the specific mechanism of the interactions requires further investigation.

Epigenetic modifications exert their function through specific enzymes, and different enzymes modify different sites or have different functions. For example, Gcn5 and CBP/P300 acetylate H3K9/H3K27 sites, Ezh2 methylates H3K27 sites, and Suv39h1 plays a role in H3K9 methylation ${ }^{13-17}$. DNMT-1 is involved in the maintenance of methylation, and DNMT3a/b functions as a de novo methyltransferase ${ }^{18,19}$. However, it remains unclear which

\footnotetext{
${ }^{1}$ Department of Clinical Laboratory; Ministry of Education Key Laboratory of Child Development and Disorders; National Clinical Research Center for Child Health and Disorders (Chongqing); China International Science and Technology Cooperation base of Child development and Critical Disorders; Children's Hospital of Chongqing Medical University, Chongqing, P.R. China. ${ }^{2}$ Department of Pediatric Research Institute, Ministry of Education Key Laboratory of Child Development and Disorders; National Clinical Research Center for Child Health and Disorders (Chongqing); China International Science and Technology Cooperation base of Child development and Critical Disorders; Children's Hospital of Chongqing Medical University, Chongqing, P.R. China. ${ }^{3}$ Department of Cardiovascular (Internal Medicine), Ministry of Education Key Laboratory of Child Development and Disorders; National Clinical Research Center for Child Health and Disorders (Chongqing); China International Science and Technology Cooperation base of Child development and Critical Disorders; Children's Hospital of Chongqing Medical University, Chongqing, P.R. China. ${ }^{4}$ Chongqing Key Laboratory of Pediatrics, Chongqing, P.R. China. ${ }^{5}$ Chengdu Women's and Children's Central Hospital, School of Medicine, University of Electronic Science and Technology of China, Chengdu, P.R. China. ${ }^{6}$ These authors contributed equally: Hao Xu and Qin Zhou. *email: jingzhu@cqmu.edu.cn
} 


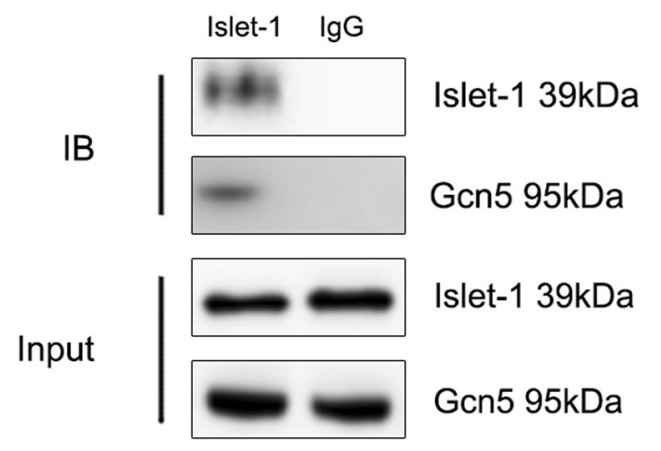

Figure 1. Co-IP experiments confirmed that islet-1 and Gcn5 bound together during MSC differentiation into cardiomyocytes induced by islet-1 overexpression. Islet-1 and IgG antibodies were chosen to pull down proteins, and the islet- 1 and Gcn 5 bands were then detected by Western blot. Gcn 5 was detected in proteins pulled down by the islet- 1 antibody, which indicated that islet-1 could form a complex with Gcn5.

specific enzymes are involved in islet-1-induced MSC differentiation into cardiomyocytes and how these enzymes interact with each other. Continuing our previous study, we will further discuss these two issues in this work.

We have confirmed that histone acetylation/methylation and DNA methylation interact with each other in the GATA4 promoter region, coregulate GATA4 expression and induce MSC differentiation into cardiomyocytes ${ }^{11}$. We also found that Gen5 and DNMT-1 play important roles in regulating GATA4 expression ${ }^{20}$. In this study, we further investigated the specific enzyme that is involved in regulating GATA4 and Nkx2.5 and the molecular mechanism of the epigenetic interaction of these two cardiac-specific transcript factor promoter regions. This research preliminarily proved the epigenetic mechanism by which MSCs differentiate into cardiomyocytes via islet- 1 and reveals the key intervention factor for further research. These findings lay the foundation for increasing MSC differentiation rates and improving the clinical application of MSCs.

\section{Results}

Islet-1 could form a complex with Gcn5 during MSC differentiation into cardiomyocytes. We transfected a lentiviral vector containing islet-1 into MSCs, and the transfection efficiency was $81 \%$ (Supplementary Figure 1a). Western blot was used to detect islet-1 expression after vector transfection (Supplementary Figure 1b). Next, we used an islet-1 antibody to pull down proteins bound to islet-1 and a Gen5 antibody to detect the existence of Gen 5 in the pulled down proteins by Western blot. Co-IP results showed that islet- 1 and Gcn 5 could form a complex after high islet-1 expression was induced in MSCs (Fig. 1). This finding confirms our previous results, which indicated that overexpression of islet- 1 could affect histone acetylation to induce MSC differentiation into cardiomyocytes ${ }^{12}$.

Gcn5 and HDAC1 are key enzymes that regulate histone acetylation levels in the GATA4/ Nkx2.5 promoter region. Our previous study showed that H3K9 acetylation in the GATA4/Nkx2.5 promoter region is associated with the expression of the two genes. In this study, we identified the key enzyme that regulates the acetylation level in the GATA4/ $\mathrm{Nkx} 2.5$ promoter region. Western blot data indicated that the level of Gcn 5 was slightly increased, and that of P300 decreased gradually during MSC differentiation into cardiomyocytes induced by islet-1 (Fig. 2a,b). ChIP-qPCR was performed to detect the binding level of Gcn5/P300 in the GATA4/Nkx2.5 promoter region. Additionally, the data showed that the binding level of Gcn 5 was increased significantly in both the GATA4 and Nkx2.5 promoter regions, but the binding level of P300 did not change significantly (Fig. 2d). After islet-1 transfection, the trend of Gen 5 binding in the GATA4/Nkx2.5 promoters was consistent with the expression of GAT4/Nkx2.5, which was detected in our previous study ${ }^{11}$. Therefore, we propose that Gcn5 is the key HAT that regulates H3K9 acetylation in the GATA4/Nkx2.5 promoter region.

Because histone acetylation is the result of the balance between HATs and HDACs, HDACs are important for histone acetylation. HDACs restore the positive charge of histones through deacetylation, increasing the attraction between DNA and histones, tightening up the loose nucleosome, and finally suppressing gene expression. Western blot analysis showed that the expression levels of HDAC2 and HDAC4 showed no significant changes, but the expression of HDAC1 gradually decreased after islet-1 transfection (Fig. 2a-c). Correspondingly, the binding level of HDAC1 in the GATA4/Nkx2.5 promoter decreased, as detected by ChIP-qPCR. However, the binding levels of HDAC2/HCAC4 were low in these regions and did not change during MSC differentiation into cardiomyocytes induced by islet-1 (Fig. 2e). These results indicate that the key HDAC that regulates H3K9 acetylation in the GATA4/Nkx2.5 promoter region is HDAC1.

G9A and DNMT-1 respectively regulated histone methylation and DNA methylation levels in the GATA4/Nkx2.5 promoter region. Histone methylation, as an important histone modification, is involved in inhibiting gene expression and heterochromatin formation. Research has reported that at the $\mathrm{H} 3 \mathrm{~K} 9$ site, HMTs interact with HATs and affect histone methylation levels in this region ${ }^{21}$. It has been indicated that histone methylation is a vital part of epigenetic modification interactions. G9A and Suv39h1 are the main enzymes involved in H3K9 methylation, and in this study, we measured these two enzymes to confirm their key role in regulating $\mathrm{H} 3 \mathrm{~K} 9$ methylation in the GATA4/Nkx2.5 promoter region. 

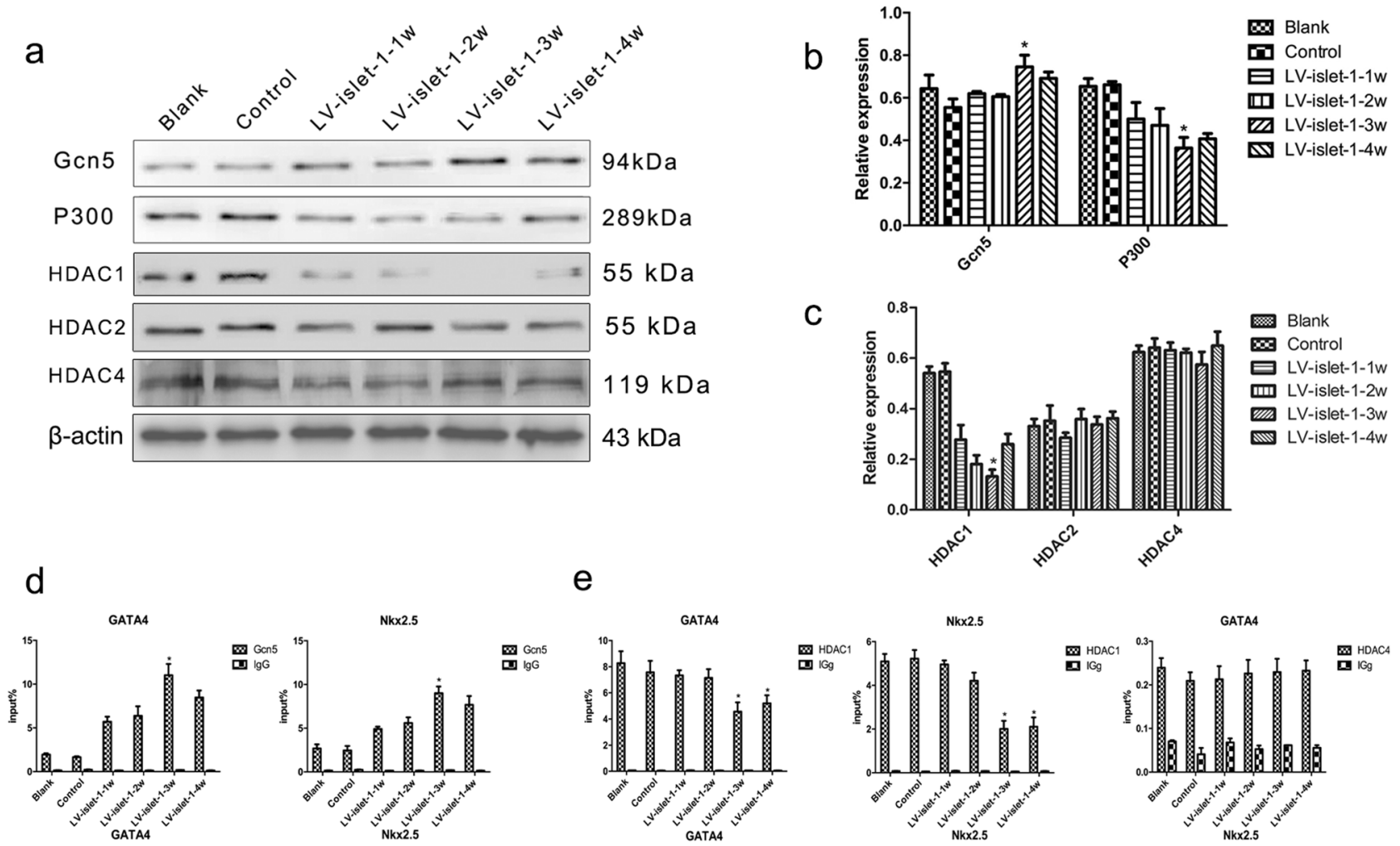

e
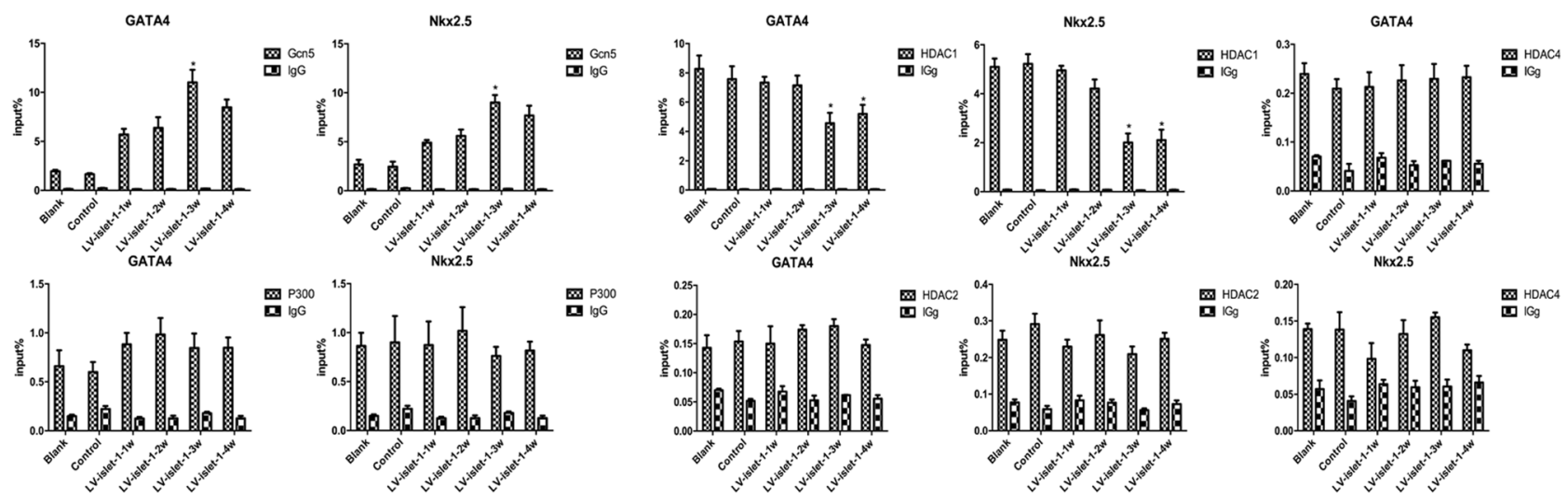

Figure 2. Identification of the key enzymes involved in $\mathrm{H} 3 \mathrm{~K} 9$ acetylation during MSC differentiation into cardiomyocytes. (a) HAT and HDAC bands were detected by Western blot, and the enzymes assessed were Gcn5, P300, HDAC1, HDAC2 and HDAC4. Images were cropped for clarity, and full-length blots/gels are presented in Supplementary Fig. 1. (b,c) Quantitative analysis of these enzymes. In the process of inducing differentiation, Gcn 5 expression slightly increased, P300 and HDAC1 expression decreased, and HDAC2 and HDAC4 expression was not obviously changed. (d,e) The binding levels of these enzymes in the GATA4/Nkx2.5 promoter were detected by ChIP-qPCR; a normal mouse IgG antibody used as a negative control. The input\% showed that the main HAT and HDAC binding in the GATA4/Nkx2.5 promoters was Gcn 5 and HDAC1, respectively. ${ }^{*} \mathrm{p}<0.05$ compared with the blank group. The error bars represent the SD of three independent experiments.

Western blot analysis was used to detect G9A/Suv39h1 expression during MSC differentiation into cardiomyocytes induced by islet-1. The results showed that the expression of G9A decreased gradually and was lowest in the LV-islet-1-3w group, but the expression of Suv39h1 did not change after islet-1 transfection compared with that in the blank and control groups (Fig. 3a,b). ChIP-qPCR indicated that in the GATA4/Nkx2.5 promoter region, the binding level of G9A was higher than that of Suv39h1; furthermore, after islet-1 transfection, the binding level of G9A in both the GATA4 and Nkx2.5 promoters decreased gradually and was lowest in the LV-islet-1-3w group, which was consistent with the H3K9 methylation level in the GATA4/Nkx2.5 promoter regions detected in our previous study. However, the binding level of Suv39h1 did not obviously change during the period of differentiation (Fig. 3d). These results suggest that G9A is the key HMT involved in regulating the histone methylation level in the GATA4/Nkx2.5 promoter region during MSC differentiation into cardiomyocytes.

DNA methylation is best known for its role in gene silencing. It can alter gene expression without changing a gene's base sequence. We used Western blot to detect the expression of DNMTs, and the results showed that the level of DNMT-1 decreased slightly and that the amount of DNMT-3a increased at the 4th week after islet-1 transfection; however, the expression of DNMT-3b was too low to detect (Fig. 3a-c). Furthermore, our ChIP-qPCR results indicated that the binding level of DNMT-1 in the GATA4/Nkx2.5 promoters was much higher than that of DNMT-3a, and the binding level of DNMT-3b was as low as that of the negative control. In addition, the binding level of DNMT-1 decreased gradually in the GATA4 promoter region during MSC differentiation into 
a

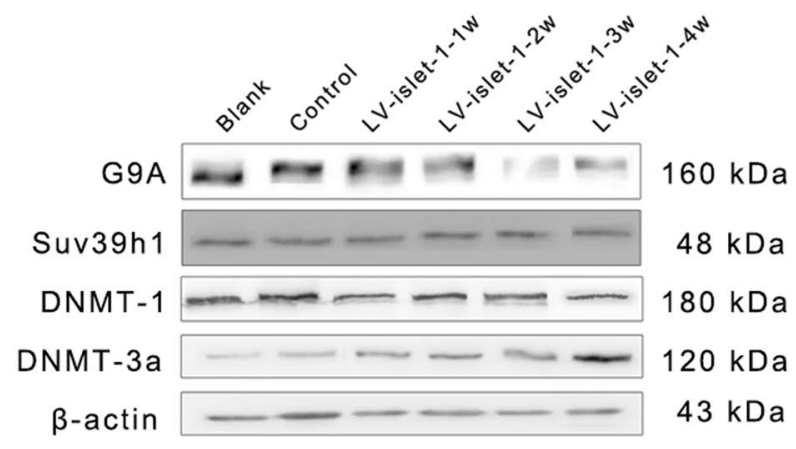

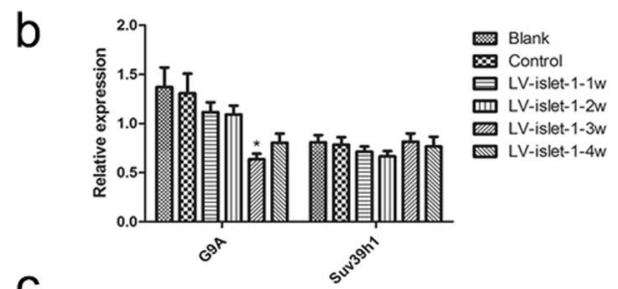

C

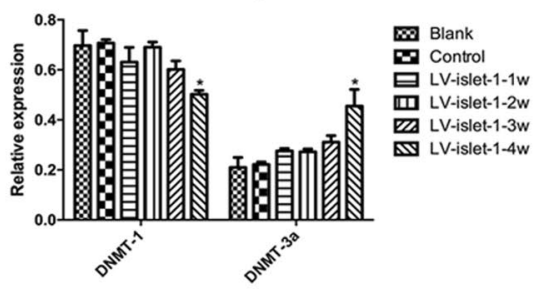

d
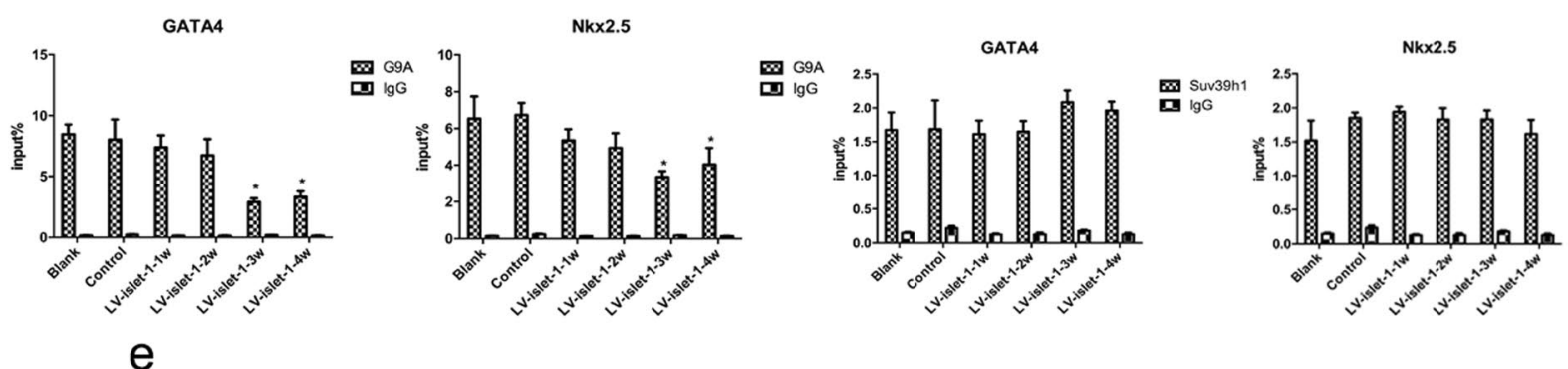

Suv39h
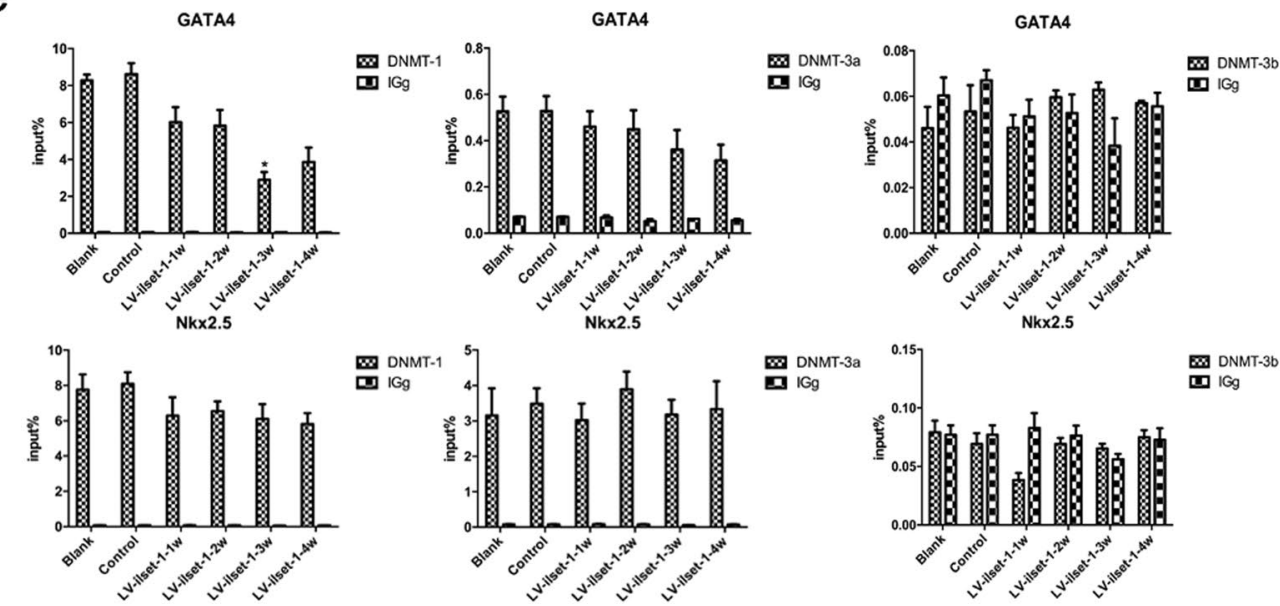

Figure 3. Identification of the key enzymes involved in histone methylation and DNA methylation. (a) HMT and DNMT bands were detected by Western blot, and the enzymes assessed were G9A, Suv39h1, DNMT-1, and DNMT-3a. Images were cropped for clarity, and full-length blots/gels are presented in Supplementary Fig. 2. (b,c) Quantitative analysis of these enzymes. In the process of inducing differentiation, G9A expression decreased gradually, DNMT-3a expression increased, and the other enzymes were not obviously changed. (d,e) The binding levels of these enzymes in the GATA4/Nkx2.5 promoter were detected by ChIP-qPCR; a normal mouse IgG antibody used as a negative control. The input\% showed that the main HMT and DNMT binding in GATA4/Nkx2.5 promoters was G9A and DNMT-1, respectively. ${ }^{*} \mathrm{p}<0.05$ compared with the blank group. The error bars represent the SD of three independent experiments.

cardiomyocytes but did not change in the Nkx2.5 promoter region (Fig. 3e). These results suggest that DNMT-1 is the key DNMT responsible for regulating the DNA methylation level in the GATA4 promoter. Additionally, the binding level of DNMT-1 did not change in the Nkx2.5 promoter region, which explains why the methylation level of the Nkx2.5 promoter was not affected by transfection with islet-1, which was found in our previous study $^{11}$.

HP1 associated with the DNA methylation level in the GATA4/Nkx2.5 promoter region. HP1 is a component of heterochromatin that recognizes and binds the methylated $\mathrm{H} 3 \mathrm{~K} 9$ site, leading to epigenetic repression. DNMT- 1 could be recruited to the methylated H3K9 site by HP1 and perform DNA methylation. Therefore, HP1 plays an important role in the interaction of histone methylation and DNA methylation. We found that the expression levels of HP1 $\alpha$ and HP1 $\beta$ were not changed during MSC differentiation into cardiomyocytes (Fig. 4a,b). However, our ChIP-qPCR results showed that the binding levels of HP1 $\alpha$ and HP1 $\beta$ 

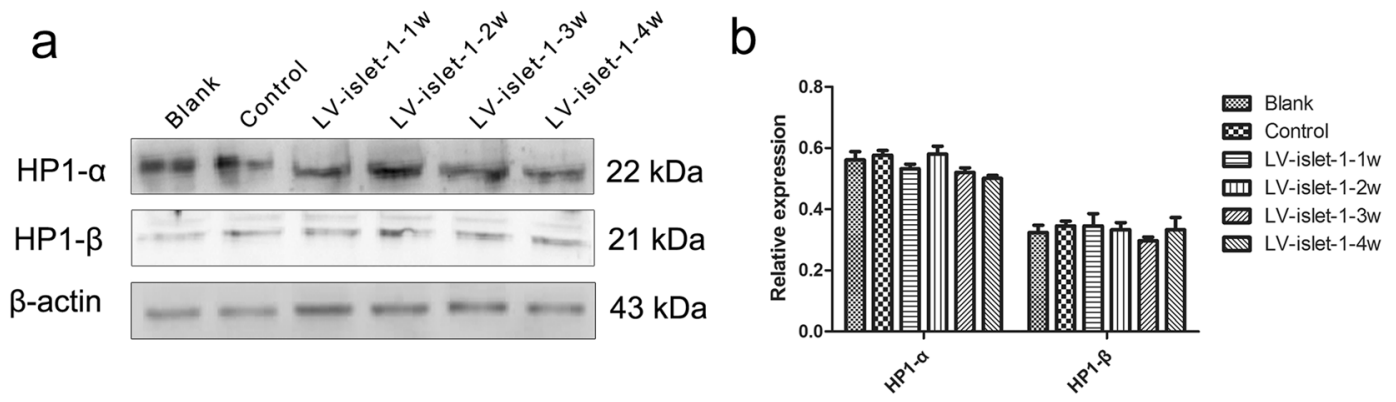

C
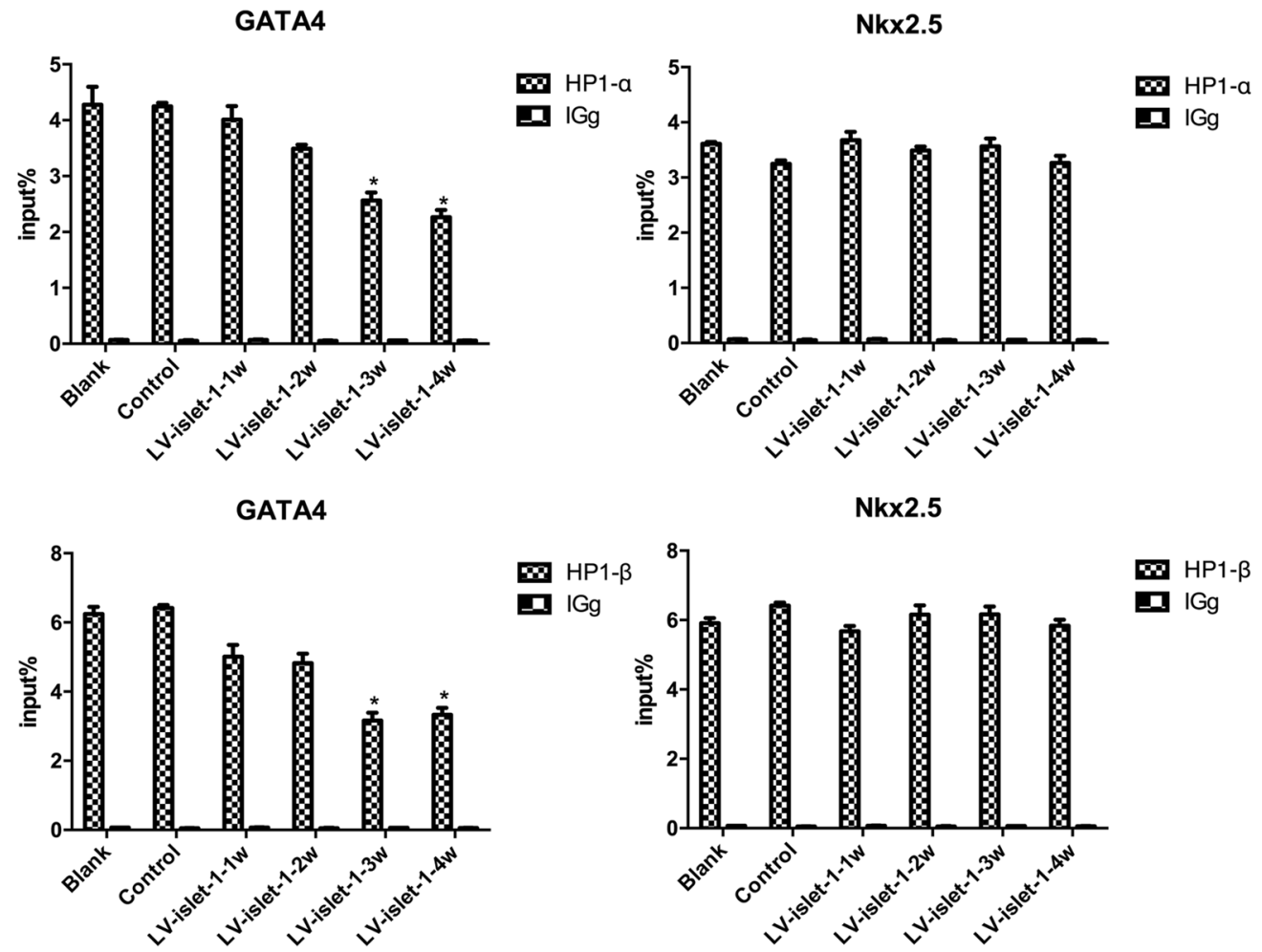

Figure 4. The expression of HP1 and its binding level in the GATA4/Nkx2.5 promoters. (a) HP1 $\alpha$ and HP1 $\beta$ bands detected by Western blot. Images were cropped for clarity, and full-length blots/gels are presented in Supplementary Fig. 3. (b) Quantitative analysis of HP1 $\alpha$ and HP1 $\beta$. In the process of inducing differentiation, HP $1 \alpha$ and HP1 $\beta$ expression was not obviously changed. (c) ChIP-qPCR detection of the binding level of HP1 $\alpha$ and HP1 $\beta$ in GATA4/Nkx2.5 promoters. A normal mouse IgG antibody was used as a negative control. The input $\%$ showed that both HP1- $\alpha$ and HP1- $\beta$ could bind to GATA4/Nkx2.5 promoters, but their binding level changed in only the GATA4 promoter. * $\mathrm{p}<0.05$ compared with the blank group. The error bars represent the $\mathrm{SD}$ of three independent experiments.

decreased in the GATA4 promoter after islet-1 transfection but did not change in the Nkx2.5 promoter (Fig. 4c). The ChIP-qPCR results were consistent with the binding levels of DNMTs. We think the reason that the DNA methylation level in the Nkx2.5 promoter was unchanged is that HP1 binding in the Nkx2.5 promoter is stable, which recruits DNMT-1 to this region and maintains DNA methylation.

Gcn 5 inhibition does not affect the expression of other key enzymes but affects their binding level in the GATA4/Nkx2.5 promoter. Of all the key enzymes that regulate GATA4/Nkx2.5 expression, Gcn5 is the only enzyme with upregulated expression and an increased binding level during MSC differentiation induced by islet-1. To confirm the interaction among the three epigenetic modifications, we inhibited Gcn5 expression and detected changes in the other key enzymes. We used the Gcn5-specific inhibitor butyrolactone 3 (MB-3) to suppress Gcn5 expression after islet-1 transfection. The Western blot results showed that the expression of Gcn 5 was significantly suppressed, but the expression of other key enzymes, HDAC1, G9A DNMT-1 and $\mathrm{HP} 1 \alpha / \beta$, was not changed in the LV-islet-1 + MB-3 group (Fig. 5a,b). This result indicates that the expression levels of these key enzymes were not affected by Gen5 inhibition. ChIP-qPCR detected the binding levels of key enzymes in the GATA4/Nkx2.5 promoters after Gcn5 inhibition. The results showed that compared with 


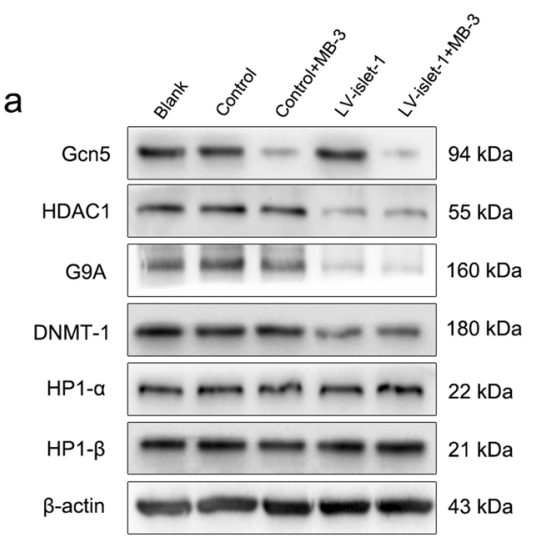

C

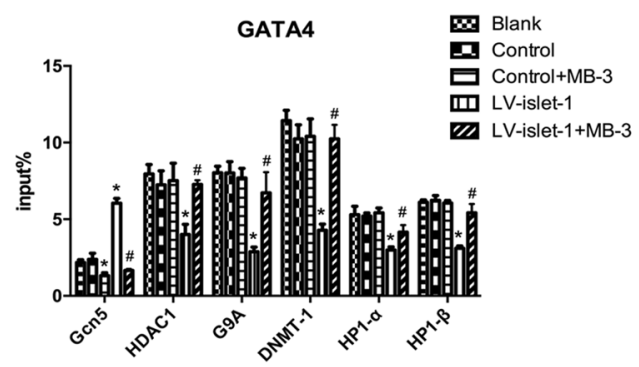

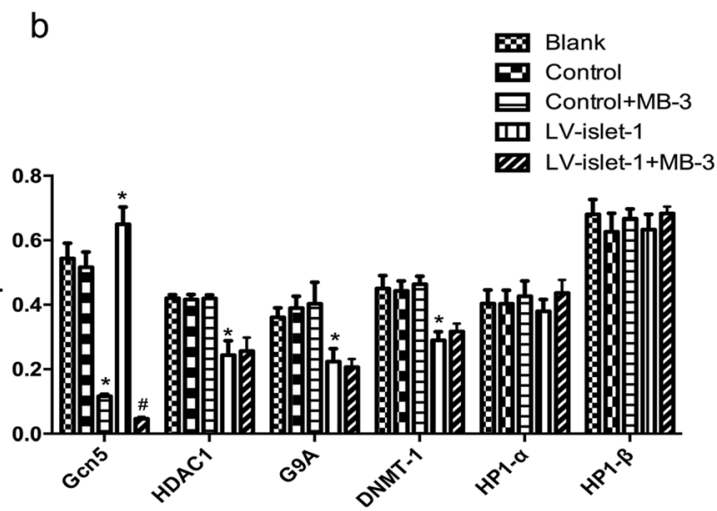

d

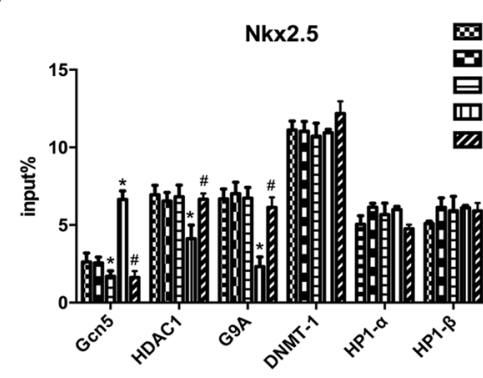

Figure 5. During MSC differentiation into cardiomyocytes induced by Islet-1, the effects of Gcn 5 inhibition on other key enzymes were assessed. (a) Western blot was used to detect the expression of the enzymes involved in regulating GATA4/Nkx2.5 after Gcn5 was inhibited by MB-3. The enzymes assessed were Gcn5, HDAC1, G9A, DNMT-1 and HP1. Images were cropped for clarity, and full-length blots/gels are presented in Supplementary Fig. 4. (b) Quantitative analysis of these enzymes. MB-3 inhibited Gcn5 expression in both the Control $+\mathrm{MB}-3$ and LV-islet-1 + MB-3 groups but had no effect on the other enzymes. (c,d) ChIP-qPCR results of the binding levels of these enzymes in the GATA4/Nkx2.5 promoters after Gcn 5 was inhibited by MB-3 during MSC differentiation. After islet- 1 transfection and Gcn 5 inhibition, the binding level of the other enzymes in the GATA4/Nkx2.5 promoters did not change compared with those in the blank, control and control $+\mathrm{MB}-3$ promoters. ${ }^{*} \mathrm{p}<0.05$ compared with the blank group, $\# \mathrm{p}<0.05$ compared with the LV-islet-1 group. The error bars represent the $\mathrm{SD}$ of three independent experiments.

that of the LV-islet-1 group, the binding level of Gcn5 was significantly decreased due to MB-3 inhibiting Gcn5 expression; as the response of Gcn5 was blocked, the binding of the other enzymes was all increased in the GATA4 promoter region (Fig. $5 \mathrm{c}$ ). In comparison, the binding levels of DNMT- 1 and HP $1 \alpha / \beta$ were not affected by Gcn 5 inhibition in the Nkx2.5 promoter region (Fig. $5 \mathrm{~d}$ ). These data suggest that $\mathrm{Gcn} 5$ interacts with these other enzymes in the GATA4 promoter region; furthermore, the fact that DNMT- 1 and HP1 $\alpha / \beta$ binding to the $\mathrm{Nkx} 2.5$ promoter was not affected by Gen 5 inhibition suggests that the interaction of these key enzymes is different in the GATA4 and Nkx2.5 promoters.

Histone acetylation/methylation and DNA methylation in the GATA4 promoter changed after Gcn5 inhibition. We investigated the effect of Gcn5 inhibition on histone acetylation/methylation and the DNA methylation level in the GATA4/Nkx2.5 promoters. The ChIP-qPCR data showed that compared with those in the LV-islet-1 group, the histone acetylation levels of the GATA4 and Nkx2.5 promoters were decreased after Gcn5 inhibition, but the histone methylation levels were increased to the levels of the blank and control groups (Fig. 6a). On the other hand, we detected the DNA methylation level of the GATA4/Nkx2.5 promoter through MSP. The results indicate that the DNA methylation level of the Nkx2.5 promoter was not affected by Gcn5 inhibition, but in the GATA4 promoter, this level was increased compared with that in the LV-islet-1 group (Fig. 6b). The BSP results showed that the DNA methylation level of the GATA4 promoter increased to $98 \%$ from $88 \%$ after Gcn5 inhibition (Fig. 6c). These results suggest that in the GATA4 promoter region, the inhibition of Gcn5 affected not only the histone acetylation level but also the histone methylation and DNA methylation levels. This is consistent with the binding level of key enzymes in the GATA4 promoter after Gcn 5 inhibition. Similarly, the DNA methylation level in the Nkx2.5 promoter did not change after Gcn5 inhibition because Gcn5 inhibition did not affect the binding of HP1 $\alpha / \beta$ and DNMT-1 to the Nkx2.5 promoter.

Islet-1 could not induce MSC differentiation into cardiomyocytes after Gcn5 inhibition. In our previous study, islet-1 overexpression promoted MSC differentiation into cardiomyocytes by increasing cardiac-specific gene (GATA4/Nkx2.5) expression. In this study, compared with that in the blank and control 
a
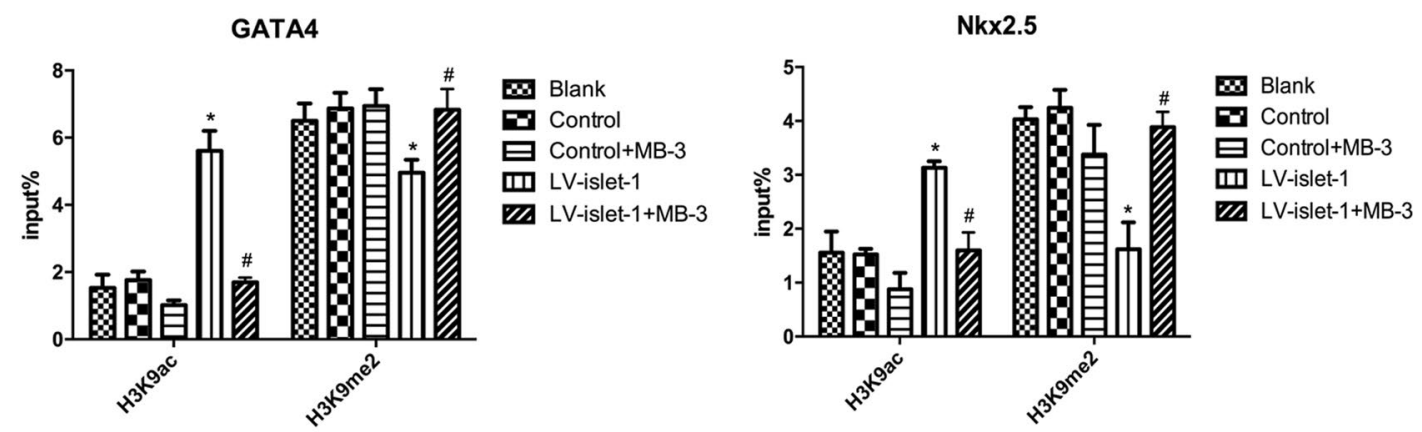

b

C
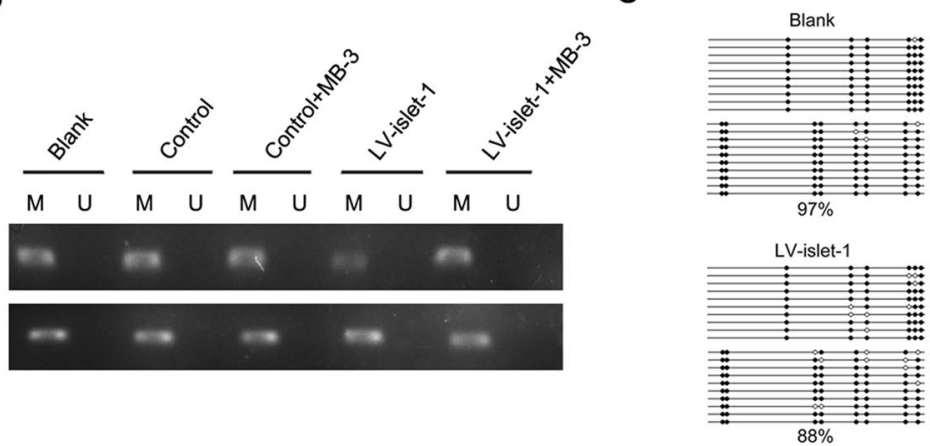

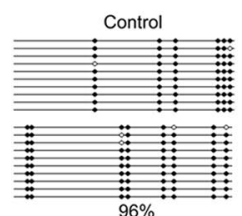

$\ddot{96 \%}$

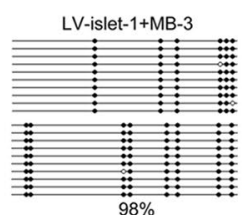

Figure 6. During MSC differentiation into cardiomyocytes induced by islet-1, changes in the levels of H3K9 acetylation/methylation and DNA methylation in the GATA4 and Nkx2.5 promoters after Gcn5 inhibition were detected. (a) ChIP-qPCR was used to detect H3K9 acetylation and methylation levels in the GATA4 and Nkx2.5 promoters. The input\% showed H3K9 acetylation and methylation levels in the LV-islet-1 + MB-3 group, in contrast to those in the LV-islet-1 group. ${ }^{*} \mathrm{p}<0.05$ compared with the blank group, ${ }^{*} \mathrm{p}<0.05$ compared with the LV-islet-1 group. The error bars represent the SD of three independent experiments. (b) MSP was used to detect the DNA methylation level in the GATA4 and Nkx2.5 promoters. Two pairs of primers were designed by Methprimer: one set of methylation-specific primers and one set of nonmethylation-specific primers. Images were cropped for clarity, and full-length blots/gels are presented in Supplementary Fig. 5. (c) Bisulfite sequencing analysis of the GATA4 promoter. Methylated CG (filled circles) and unmethylated CG (open circles) are indicated. The methylation rate of the GATA4 promoter was $88 \%$ in the LV-islet-1 group and $98 \%$ in the LVislet-1+MB-3 group, which were the same as those in the blank and control groups.

groups, the expression of GATA4/Nkx2.5 was not changed after Gcn5 inhibition, even when islet- 1 was overexpressed (Fig. 7a). We thought islet-1 could induce MSC differentiation into cardiomyocytes because MSCs began to express the cardiac-specific gene cTnT after islet-1 transfection, and the myocardium electrophysiological properties of MSCs may be related to Cx43 expression. However, through immunofluorescence, we found that after Gcn5 inhibition, islet-1 could not induce MSC expression of cTnT and Cx43 (Fig. 7b). These results suggest that MSCs could not be induced into cardiomyocytes after Gcn5 inhibition, even in the presence of islet-1.

\section{Discussion}

Epigenetic manipulation to differentiate MSCs into specialized cells has the potential to serve regenerative purposes. Various studies support the involvement of epigenetic mechanisms through gene expression control, stem cell self-renewal and lineage fate determination ${ }^{22,23}$. During differentiation into a particular lineage, the specific genes of this lineage undergo active transcription, and the genes responsible for self-renewal and pluripotency are repressed $^{22,24}$. This on-off mechanism is associated with posttranslational modifications, especially histone acetylation/methylation and promoter DNA methylation. These epigenetic modifications are critical for regulating gene expression, and many studies have focused on one of them. However, in practice, regulating gene expression is extensively coordinated by a network of many factors working together ${ }^{21,25-27}$, which is far beyond the capability of a single epigenetic modification. In the complicated differentiation process, regulating gene expression may be more involved. Different epigenetic modifications probably regulate the same gene through interactions with each other $^{28-30}$. Regarding MSC differentiation, it will be helpful to investigate the mechanism of interactions between epigenetic modifications.

Our previous study reported that histone acetylation/methylation and DNA methylation are involved in MSC differentiation into cardiomyocytes by regulating GATA4 and Nkx2.5 expression, and these modifications showed obvious interactions with each other in the GATA4/Nkx2.5 promoter. DNA methylation was not involved in regulating Nkx2.5 expression and did not interact with the other two modifications in the $\mathrm{Nkx} 2.5$ promoter ${ }^{11}$. 
a

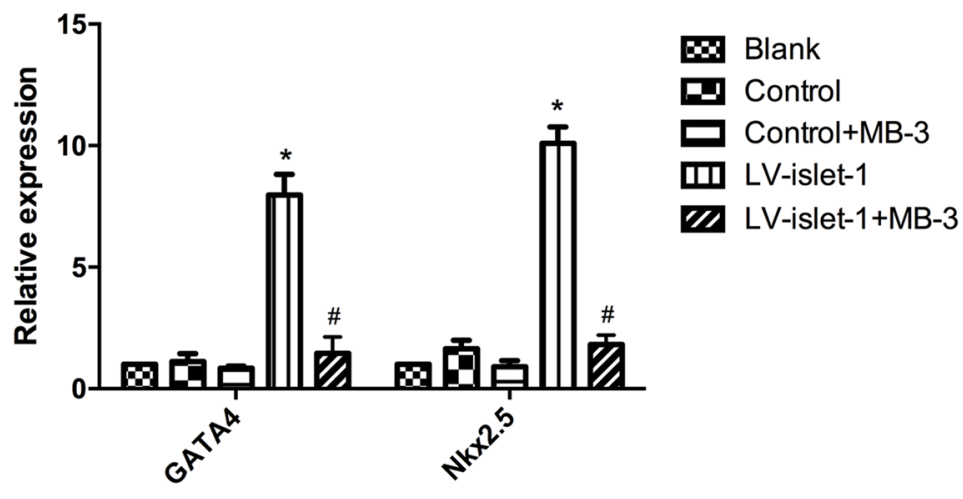

b
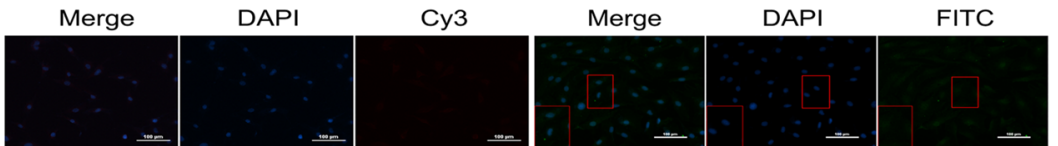

Control+MB-3
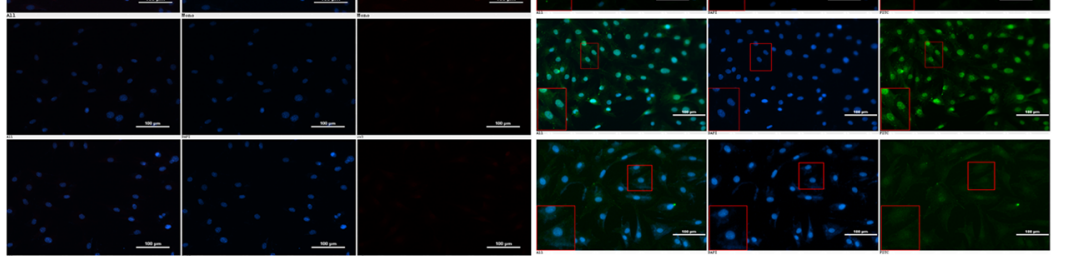

LV-islet-1
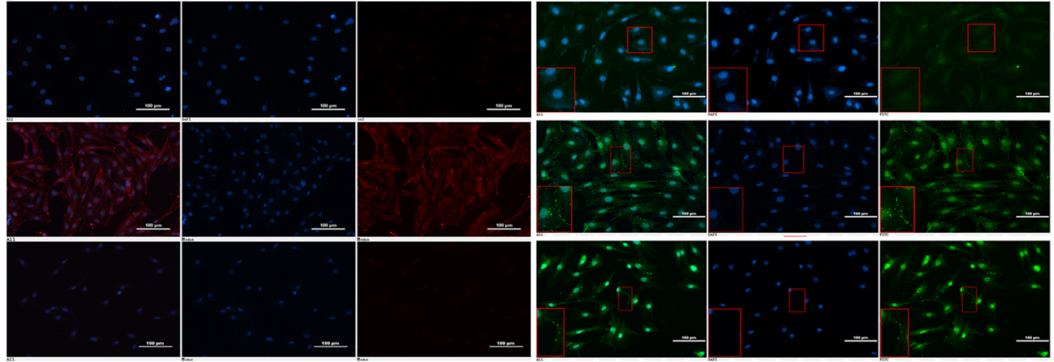

LV-islet-1+MB-3

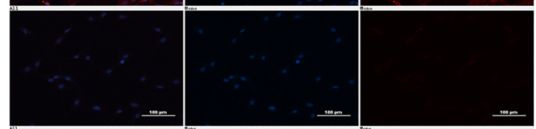

Figure 7. The effect of Gen 5 inhibition on MSC differentiation into cardiomyocytes induced by islet-1. (a) FQ-PCR detected the expression levels of GATA4 and Nkx2.5 after Gcn5 was inhibited. GATA4 and Nkx2.5 expression increased after islet- 1 transfection, but in the LV-islet-1 + MB-3 group, their expression did not change compared with that in the blank and control groups. ${ }^{*} \mathrm{p}<0.05$ compared with the blank group, ${ }^{*} \mathrm{p}<0.05$ compared with the LV-islet-1 group. The error bars represent the SD of three independent experiments. (b) An immunofluorescence assay was used to detect the expression of cTnT and Cx43 (100×). The fluorescent dye of cTnT was CY3, that of $\mathrm{Cx} 43$ was FITC, and DAPI was used for nuclear staining. cTnT express in Cytoplasm, and only expressed in LV-islet-1 group; Cx43 express mainly in Membrane, and expressed much more in LV-islet-1 group than other groups. The block in the lower left corner of a single image is the local amplification $(200 \times)$ of the corresponding positions.

The interactions among histone acetylation, histone methylation and DNA methylation work in different ways in the GATA4 and Nkx2.5 promoters, but the details are not completely understood. GATA4 and Nkx2.5 are critical transcription factors in heart development, and elucidating the molecular mechanism will contribute to regulating their expression and help in the development of a method to differentiate MSCs into cardiomyocytes.

In this study, we found that the key enzymes of $\mathrm{H} 3 \mathrm{~K} 9$ acetylation, $\mathrm{H} 3 \mathrm{~K} 9$ methylation and DNA methylation in the GATA4/Nkx2.5 promoters are Gcn5/HDAC1, G9A and DNMT-1, respectively. During the process of MSC differentiation induced by islet-1, the binding level of Gen 5 in the promoter region of GATA4/Nkx2.5 was obviously increased. On the other hand, the binding levels of G9A, DNMT-1, and HDAC1 were all decreased in the GATA4 promoter, but that of DNMT-1 in the Nkx2.5 promoter was not changed. When the cells were treated with the Gcn5 inhibitor MB-3, the expression levels of other key enzymes (HDAC1, G9A, and DNMT-1) were unaffected, and their binding levels in the GATA4/Nkx2.5 promoters were also unchanged. This indicated that the changes in these key enzymes were not caused by islet- 1 but by Gcn 5 interacting with them. There is another critical factor involved in the interactions among these key enzymes, HP1 ${ }^{31-33}$. HP1 can bind to the methylated H3K9 site and recruit DNMT-1 binding to this region; thus, the CpG sites of this region are methylated ${ }^{34-36}$. When MSCs differentiated into cardiomyocytes, the H3K9 methylation level in the GATA4 promoter was decreased, and the binding of HP1 to this region was reduced as well. However, HP1 binding to the Nkx2.5 promoter was unaffected, and even the H3K9 methylation level of this region was decreased. This could be the reason why DNMT-1 binding to the Nkx2.5 promoter was unaffected during MSC differentiation.

In our study, when islet- 1 was used to induce MSC differentiation into cardiomyocytes, Gcn 5 expression was slightly increased, and its binding level in the GATA4 promoter was significantly increased. On the other hand, research has reported that Gcn5 and islet-1 could form a complex ${ }^{37}$. Therefore, we thought that islet-1 


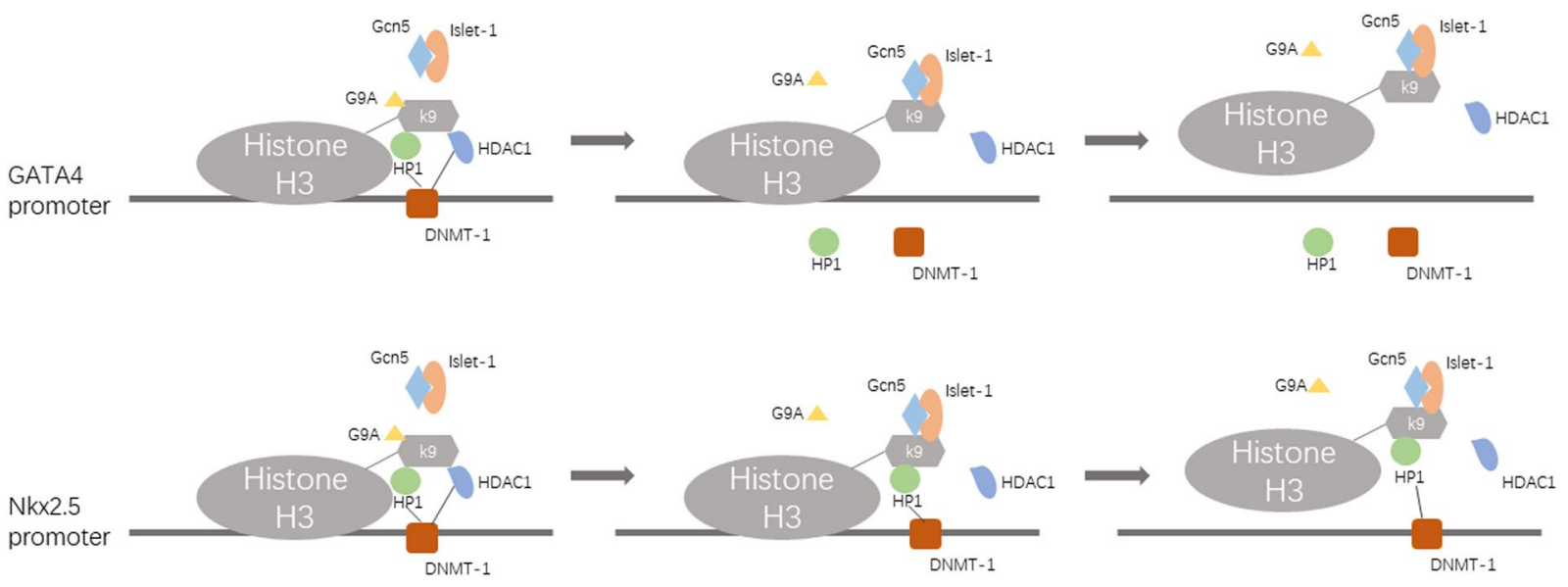

Figure 8. The epigenetic modification model of MSC differentiation into cardiomyocytes induced by islet1. Islet-1 guides Gcn 5 binding to the GATA4 and Nkx2.5 promoters, and Gcn5 interacts with G9A, HP1, DNMT- 1 and HDAC1. Then, the chromatin of the GATA4 and Nkx2.5 promoter region loosened because of the hyperacetylation of these regions, which is conducive to GATA 4 and Nkx2.5 expression.

could increase GATA4/Nkx2.5 expression by combining with Gcn5 and guiding it to the GATA4/Nkx2.5 promoters instead of by increasing Gcn5 expression. Based on the above results, we propose an epigenetic modification model of MSC differentiation into cardiomyocytes induced by islet-1 (Fig. 8). First, the overexpressed islet- 1 could guide more Gcn 5 to bind to the GATA4 promoter; then, the $\mathrm{H} 3 \mathrm{~K} 9$ site of this region may be hyperacetylated. After that, as more Gcn 5 binds to the GATA4 promoter, more G9A is replaced, and the $\mathrm{H} 3 \mathrm{~K} 9$ site becomes hypomethylated. Then, the binding level of HP1 to the GATA4 promoter decreases because of $\mathrm{H} 3 \mathrm{~K} 9$ hypomethylation in this region, which simultaneously frees DNMT-1 from the GATA4 promoter, and the CpG methylation level of this region decreases. Furthermore, HDAC1 is replaced by Gcn 5 from the H3K9 site of the GATA4 promoter. However, according to the literature, DNMT-1 can recruit HDAC1. Therefore, HDAC1 binding to the GATA4 promoter further decreases because DNMT-1 is lost from this region. This phenomenon further increases the H3K9 acetylation level of the GATA4 promoter. Finally, the chromatin structure of the GATA4 promoter region becomes loose, which is conducive to GATA4 expression. The interactions among these key enzymes in the Nkx2.5 promoter is different from those in the GATA4 promoter. HP1 binding to the Nkx 2.5 promoter is unaffected by the $\mathrm{H} 3 \mathrm{~K} 9$ methylation level of this region, which ensures that DNMT-1 binds to this region stably. In the process of induced differentiation, we found significant differences in some parameters, such as the binding levels of Gcn 5 and DNMT-1 in the GATA4/Nkx2.5 promoters, which were lost at the 4th week; at this time point, we also found that the protein expression of islet-1 decreased (Supplementary Figure 2), we were not sure whether these changes were associated with the reduction of Islet- 1 . We thought that it may be a trend of differentiation progress, and the specific meanings of these changes require our further study.

Our results showed that when Gcn 5 was inhibited, the interactions among these key enzymes were blocked, the expression of GATA4 and Nkx2.5 was repressed, and islet-1 could not induce MSC differentiation into cardiomyocytes. These results indicated that the epigenetic modifications and the interactions among these key enzymes are critical for MSC differentiation. However, in practice, the mechanism of MSC differentiation is more complicated. For example, in addition to epigenetic modifications, the stemness of MSCs and the energy metabolism conversion during MSC differentiation are equally important. By studying these two topics, we can thoroughly understand the mechanism of MSC differentiation.

\section{Methods}

Cell culture, lentiviral vector transfection and MB-3 treatment. C3H10T1/2 cells (University of Chicago Molecular Oncology Laboratory, Chicago, IL, USA) were cultured in DMEM supplemented with 10\% foetal bovine serum (FBS; Millipore, USA), lentiviral vectors containing islet-1 or GFP (MOI=20, GENECHEM, Shanghai, China) and $5 \mu \mathrm{g} / \mathrm{ml}$ polybrene. The culture medium was replaced after $24 \mathrm{~h}$ of incubation at $37^{\circ} \mathrm{C}$ in $5 \%$ $\mathrm{CO} 2$. The cells were divided into three groups: a blank group (C3H10T1/2), a control group (lentiviral vector with GFP), and an LV-islet-1 group (lentiviral vector with islet-1). According to the transfection duration, the LV-islet-1 group was further divided into four subgroups: LV-islet-1-1w, LV-islet-1-2w, LV-islet-1-3w, and LV-islet-1-4w. Butyrolactone 3 (MB-3, $100 \mu \mathrm{mol}$, Santa Cruz, sc-358657) was added to the culture medium after LV-islet-1 or GFP transfection for $24 \mathrm{~h}$; the treatment was applied for 4 weeks, and the cells were defined as the LV-islet- $1+\mathrm{MB}-3$ group and the control + MB-3 group. The culture medium of all groups was changed every 2-3 days.

Co-immunoprecipitation (Co-IP) assay. Co-immunoprecipitation was conducted using a Co-IP assay kit (Merck Millipore, DA, Germany), and the total protein concentrations of the cell lysates were measured with a BCA assay. An anti-islet-1 (Abcam, Cambridge, MA, UK) antibody was used to pull down islet-1, and an anti-IgG antibody was used as a negative control. Protein collection was analysed by Western blot using an anti-Gen 5 (Epigentek, Farmingdale, NY, USA) antibody to detect its existence in islet-1-recruiting proteins. 
Chromatin immunoprecipitation (ChIP)-qPCR assay. Cells were resuspended in PBS; formaldehyde (1\%) was added to the samples to crosslink protein-DNA complexes for $5 \mathrm{~min}$ at room temperature, and glycine was then added to a final concentration of $0.125 \mathrm{M}$ for $5 \mathrm{~min}$ to terminate the crosslinking. The crosslinked material was fragmented by sonication (UCD-200, Bioruptor), which consisted of 25 cycles of $30 \mathrm{~s}$ each time with 30-s cooldown intervals. ChIP was performed using a ChIP assay kit (Merck Millipore, DA, GER), and the antibodies used are listed in Supplementary Table 1. The experiment had both a positive control group (precipitated with an anti-RNA polymerase II antibody) and a negative control group (precipitated with normal mouse IgG); $1 \%$ of the starting chromatin was used as an input. The primers were designed as follows: Nkx2.5 (forward) 5'-ACCGCCTGGGTGATAGAC-3', Nkx2.5 (reverse) 5'-CCCTCCCGAGATTGAAGAT-3', GATA4 (forward) 5'-GCTACAGGGAGTGATGAGAAG-3', and GATA4 (reverse) $5^{\prime}$-CACCAGCCCAGGAGTTTAT- $3^{\prime}$. The qPCR reaction settings were as follows: step $1,95^{\circ} \mathrm{C}$ for $5 \mathrm{~min}$; step 2,40 cycles of $95^{\circ} \mathrm{C}$ for $15 \mathrm{~s}$ and $60^{\circ} \mathrm{C}$ for $30 \mathrm{~s}$; step 3 , $65^{\circ} \mathrm{C}$ for $5 \mathrm{~s}$ and $95^{\circ} \mathrm{C}$ for $0.5 \mathrm{~s}$. To calculate the input $\%$, the following equation was used: $\mathrm{Ct}(\mathrm{input})-6.644$; then, the input $\%$ was adjusted to $100 \%$ as follows: $100 * 2 \wedge$ (Adjusted Ct (input) - Ct (IP)).

Protein extraction and western blot. Proteins were extracted from cells using RIPA Reagent (P0013B, Beyotime Biotech, China). Protein samples were mixed with $5 \times$ buffer and boiled for 5 min before being loaded onto a $10 \%$ SDS-PAGE gel. After electrophoresis, the proteins were transferred to polyvinylidene fluoride (PVDF) membranes (Millipore, USA). According to the markers, the membranes were cut into pieces and incubated in $5 \%$ nonfat milk-PBST for $1 \mathrm{~h}$. The membranes were incubated with primary antibodies (Supplementary Table 1) overnight at $4{ }^{\circ} \mathrm{C}$ and then washed with PBST 3 times for $10 \mathrm{~min}$. After that, the membranes were incubated with the corresponding secondary antibody. Positive bands were detected by chemiluminescent reactions (Millipore, USA).

Bisulfite sequencing PCR (BSP) assay. DNA was extracted from cells using a DNA extraction kit (AP-MN-MS-GDNA-250, Axygen) and then treated with bisulfite (ZYMO RESEARCHA, California, USA). The PCR products were extracted from the gel (AP-GX-250, Axygen) and ligated into the pMD ${ }^{\circledR} 18-\mathrm{T}$ Vector (D101B, TAKARA). Plasmid-transformed DH5 $\alpha$ bacteria were cultured overnight, and plasmid DNA was isolated (Axygen). At least 10 separate clones were chosen for sequence analysis.

Genomic DNA extraction and methylation-specific PCR (MSP). Genomic DNA was collected using a genomic DNA purification kit (TIANGEN, Beijing, China) and modified using an EZ DNA Methylation-Gold Kit (ZYMO RESEARCHA, California, USA). The primers for methylation-specific PCR (MS-PCR) were designed as follows: methylation-specific primers, Nkx2.5 (forward) 5'-ATTAGGTGACGTAGAATTGTTCGTC-3', Nkx2.5 (reverse) 5'-CGCCTCTCTACCCTAAATATAACG-3'; GATA4 (forward) 5'-GGGTTTATAGGTATTGAC GTCGA-3' ${ }^{\prime}$, and GATA4 (reverse) $5^{\prime}$-GATAAAAACTACAAAACGCCGAA- $3^{\prime}$. The nonmethylation-specific primers were Nkx2.5 (forward) primer 5'-TAGGTGATGTAGAATTGTTTGTTGT-3', Nkx2.5 (reverse) primer 5'-CCACCTCTCTACCCTAAATATAACAC-3'; GATA4 (forward) 5'-AGGGTTTATAGGTATTGATGTTGA-3', and GATA4 (reverse) $5^{\prime}$-CCAATAAAAACTACAAAACACCAAA- $3^{\prime}$. The PCR products were run on a $2 \%$ agarose gel, stained with ethidium bromide, and evaluated under UV light.

Total RNA extraction and real-time PCR. The total RNA samples were collected using an RNA extraction kit (RP120; BioTeke) and transformed into cDNA using a PrimeScript RT reagent kit (Takara, Dalian, Liaoning, China). Then, the resulting cDNA samples were amplified with gene-specific primers and a SYBR Green dye kit (Takara, Dalian, Liaoning, China). Primers were designed as follows: Nkx2.5 (forward) 5'-GAGCCTGGTAGGGAAAGAGC-3', Nkx2.5 (reverse) 5'-GGTGGGTGTGAAATCTGAGG-3', GATA4 (forward) $5^{\prime}$-GACTACCACCACCACGCTGT-3', and GATA4 (reverse) 5'-ATTCAGGTTCTTGGGCTTCC-3'.

Immunofluorescence. The cells were fixed in $4{ }^{\circ} \mathrm{C}$ acetone for $15 \mathrm{~min}$ and blocked with goat serum (1:20). Then, primary anti-cardiac troponin T monoclonal (1:400, Abcam) and anti-connexin 43 polyclonal (1:100, Abcam) antibodies were added for overnight incubation at $4{ }^{\circ} \mathrm{C}$. Next, secondary antibodies (1:150; CoWin Bioscience, Beijing, China) conjugated to $\mathrm{Cy} 3$ were added and incubated for $1 \mathrm{~h}$ at $37^{\circ} \mathrm{C}$. DAPI was then added for $3 \mathrm{~min}$. Images were acquired under a fluorescence microscope (BX51; Olympus).

Statistical analysis. Each experiment was repeated at least three times. All data are expressed as the means $\pm S D$, while statistical evaluations were performed using independent samples and t-tests, continuity correction chi-square tests and one-way ANOVA. SPSS 17.0 software (SPSS Inc., Armonk, NY, USA) was used for statistical analysis. A value of $\mathrm{P}<0.05$ was considered statistically significant for all analyses.

Data availability

All data used during the current study available from the corresponding author on reasonable request.

Received: 12 June 2019; Accepted: 22 November 2019;

Published online: 04 February 2020

\section{References}

1. Bianco, P., Robey, P. G. \& Simmons, P. J. Mesenchymal stem cells: revisiting history, concepts, and assays. Cell. Stem. Cell. 2(4), 313-9 (2008).

2. Kumar, S. L. S. D. \& Mok, S. PL. Cellular Reparative Mechanisms of Mesenchymal Stem Cells for Retinal Diseases. Int. J. Mol. Sci. 18(8), 1406, https://doi.org/10.3390/ijms18081406 (2017). 
3. Fahy, N., Alini, M. \& Stoddart, M. J. Mechanical stimulation of mesenchymal stem cells: Implications for cartilage tissue engineering. J. Orthop. Res. Jan. 36(1), 52-63 (2018).

4. EEM, C. et al. Knockdown of SIRT7 enhances the osteogenic differentiation of human bone marrow mesenchymal stem cells partly via activation of the Wnt/3-catenin signaling pathway. Cell Death Dis. 8(9), e3042, https://doi.org/10.1038/cddis.2017.429 (2017).

5. Chou, S. H. et al. Mesenchymal stem cell insights: prospects in cardiovascular therapy. Cell Transplant. 23(4-5), 513-29 (2014).

6. Majka, M., Sułkowski, M., Badyra, B. \& Musiałek, P. Concise Review: Mesenchymal Stem Cells in Cardiovascular Regeneration: Emerging Research Directions and Clinical Applications. Stem Cell Transl. Med. 6(10), 1859-1867 (2017).

7. Khajeniazi, S., Solati, M., Yazdani, Y., Soleimani, M. \& Kianmehr, A. Synergistic induction of cardiomyocyte differentiation from human bone marrow mesenchymal stem cells by interleukin $1 \beta$ and 5-azacytidine. Biol. Chem. 397(12), 1355-1364 (2016).

8. Zhang, Y., Chu, Y., Shen, W. \& Dou, Z. Effect of 5-azacytidine induction duration on differentiation of human first-trimester fetal mesenchymal stem cells towards cardiomyocyte-like cells. Interact. Cardiovasc. Thorac. Surg. 9(6), 943-6 (2009).

9. Yang, G. et al. Trichostatin a promotes cardiomyocyte differentiation of rat mesenchymal stem cells after 5-azacytidine induction or during coculture with neonatal cardiomyocytes via a mechanism independent of histone deacetylase inhibition. Cell Transplant. 21(5), 985-96 (2012).

10. Huang, Y. S. et al. Mesenchymal stem cells from rat olfactory bulbs can differentiate into cells with cardiomyocyte characteristics. J. Tissue Eng. Regen. Med. 9(12), E191-201 (2015).

11. Xu, H. et al. Histone modifications interact with DNA methylation at the GATA4 promoter during differentiation of mesenchymal stem cells into cardiomyocyte-like cells. Cell Prolif. Jun. 49(3), 315-29 (2016).

12. Yin, N. et al. Islet-1 promotes the cardiac-specific differentiation of mesenchymal stem cells through the regulation of histone acetylation. Int. J. Mol. Med. 33(5), 1075-82 (2014).

13. Huang, B., Li, G. \& Jiang, X. H. Fate determination in mesenchymal stem cells: a perspective from histone-modifying enzymes. Stem Cell Res. Ther. 6, 35 (2015).

14. Marmorstein, R. \& Trievel, R. C. Histone modifying enzymes: structures, mechanisms, and specificities. Biochim. Biophys. Acta. 1789(1), 58-68 (2009).

15. Wang, M., Yu, Q., Wang, L. \& Gu, H. Distinct patterns of histone modifications at cardiac-specific gene promoters between cardiac stem cells and mesenchymal stem cells. Am. J. Physiol. Cell Physiol. 304(11), C1080-90 (2013).

16. Wei, Y. et al. CDK1-dependent phosphorylation of EZH2 suppresses methylation of H3K27 and promotes osteogenic differentiation of human mesenchymal stem cells. Nat. Cell Biol. 13(1), 87-94 (2011).

17. Aoyama, T. et al. Histone modifiers, YY1 and p300, regulate the expression of cartilage-specific gene, chondromodulin-I, in mesenchymal stem cells. J. Biol. Chem. 285(39), 29842-50 (2010).

18. Bird, A. DNA methylation patterns and epigenetic memory. Genes. Dev. 16(1), 6-21 (2002).

19. Moore, L. D., Le, T. \& Fan, G. DNA methylation and its basic function. Neuropsychopharmacology. 38(1), 23-38 (2013).

20. Yi, Q. et al. Islet-1 induces the differentiation of mesenchymal stem cells into cardiomyocyte-like cells through the regulation of Gcn5 and DNMT-1. Mol. Med. Rep. May. 15(5), 2511-2520 (2017).

21. $\mathrm{Wu}, \mathrm{L}$. P. et al. Histone deacetylase inhibitor depsipeptide activates silenced genes through decreasing both CpG and H3K9 methylation on the promoter. Mol. Cell Biol. 28(10), 3219-35 (2008).

22. Saidi, N. et al. Dynamic changes of epigenetic signatures during chondrogenic and adipogenic differentiation of mesenchymal stem cells. Biomed. Pharmacother. 89, 719-731 (2017).

23. Vincent, A. \& Van Seuningen, I. Epigenetics, stem cells and epithelial cell fate. Differentiation. 78(2-3), 99-107 (2009).

24. Kouzarides, T. Chromatin modifications and their function. Cell. 128(4), 693-705 (2007).

25. Qiu, J. Epigenetics: unfinished symphony. Nature. 441(7090), 143-5 (2006).

26. Berger, S. L. The complex language of chromatin regulation during transcription. Nature. 447(7143), 407-12 (2007).

27. Fuks, F. DNA methylation and histone modifications: teaming up to silence genes. Curr. Opin. Genet. Dev. 15(5), 490-5 (2005).

28. Ghoshal, K. et al. Inhibitors of histone deacetylase and DNA methyltransferase synergistically activate the methylated metallothionein I promoter by activating the transcription factor MTF-1 and forming an open chromatin structure. Mol. Cell Biol. 22(23), 8302-19 (2002).

29. Bachman, K. E. et al. Histone modifications and silencing prior to DNA methylation of a tumor suppressor gene. Cancer Cell. 3(1), 89-95 (2003).

30. Strunnikova, M. et al. Chromatin inactivation precedes de novo DNA methylation during the progressive epigenetic silencing of the RASSF1A promoter. Mol. Cell Biol. 25(10), 3923-33 (2005).

31. Lehnertz, B. et al. Suv39h-mediated histone H3 lysine 9 methylation directs DNA methylation to major satellite repeats at pericentric heterochromatin. Curr. Biol. 13(14), 1192-200 (2003).

32. Bannister, A. J. et al. Selective recognition of methylated lysine 9 on histone H3 by the HP1 chromo domain. Nature. 410(6824), $120-4(2001)$.

33. Lachner, M., O'Carroll, D., Rea, S., Mechtler, K. \& Jenuwein, T. Methylation of histone H3 lysine 9 creates a binding site for HP1 proteins. Nature. 410(6824), 116-20 (2001).

34. Smallwood, A., Estève, P. O., Pradhan, S. \& Carey, M. Functional cooperation between HP1 and DNMT1 mediates gene silencing. Genes. Dev. 21(10), 1169-78 (2007).

35. Fuks, F., Hurd, P. J., Deplus, R. \& Kouzarides, T. The DNA methyltransferases associate with HP1 and the SUV39H1 histone methyltransferase. Nucleic Acids Res. 31(9), 2305-12 (2003).

36. Ma, Y. et al. DNA CpG hypomethylation induces heterochromatin reorganization involving the histone variant macroH2A. J. Cell Sci. 118(Pt 8), 1607-16 (2005).

37. Na Zhou et al. Screening Differentiation Related GCN5 Recruitment Proteins during Differentiation of Mesenchymal Stem Cells into Cardiac Like Myocytes. Chinese. J. Cell Biol. 32(01), 61-68 (2010).

\section{Acknowledgements}

This work was supported by the National Natural Science Foundation of China (Grant Number 81670270 \& Grant Number 81700250), and Chongqing Science \& Technology Commission (Grant Number cstc2016jcyjA0420).

\section{Author contributions}

Jing Zhu and Jie Tian conceived and designed the project. Hao Xu and Qin Zhou carried out the experiments and drafted the manuscript, Bin Tan contributed to edit the figures. Qin Yi performed statistical analyses. Xueni Chen carried out the sample collection. Yue Wang and Xia Yu contributed to cells culture, reagent procurement and management.

\section{Competing interests}

The authors declare no competing interests. 


\section{Additional information}

Supplementary information is available for this paper at https://doi.org/10.1038/s41598-020-58387-8.

Correspondence and requests for materials should be addressed to J.Z.

Reprints and permissions information is available at www.nature.com/reprints.

Publisher's note Springer Nature remains neutral with regard to jurisdictional claims in published maps and institutional affiliations.

(c) (i) Open Access This article is licensed under a Creative Commons Attribution 4.0 International License, which permits use, sharing, adaptation, distribution and reproduction in any medium or format, as long as you give appropriate credit to the original author(s) and the source, provide a link to the Creative Commons license, and indicate if changes were made. The images or other third party material in this article are included in the article's Creative Commons license, unless indicated otherwise in a credit line to the material. If material is not included in the article's Creative Commons license and your intended use is not permitted by statutory regulation or exceeds the permitted use, you will need to obtain permission directly from the copyright holder. To view a copy of this license, visit http://creativecommons.org/licenses/by/4.0/.

(C) The Author(s) 2020 